\title{
Reply to the comments on our article "The fate of patellar tendon and infrapatellar fat pad after arthroscopy via central portal"
}

\author{
Ahmet Bayar \\ Received: 16 November 2008/ Accepted: 20 January 2009/Published online: 9 April 2009 \\ (C) Springer-Verlag 2009
}

Dear Editor,

We are pleased to read that more experience is being shared about effects of Central Patellar (CP) portal on patellar tendon, which was somehow an ignored topic in arthroscopic surgery until recently. We thank Dr Checa for this important contribution and comments on our article [1], although these two studies are different in terms of methodology.

We were also surprised to read about their study mentioned in the letter, in which no echogenic abnormalities or thickening in the sagittal diameter of the tendon was observed four to 6 weeks after arthroscopy via CP portal. The authors had performed the arthroscopies for buckethandle tears of medial meniscus, which was also the indication for the patients in our series. They stated that the procedure was well tolerated by the majority of patients and this finding was also similar to our study. None of our patients complained of anterior knee pain or had positive clinical examination signs of patellofemoral joint pathology. However, our radiological findings were different. Most of the tendons in our study demonstrated the radiological sequela of the procedure about a mean of 28 months after arthroscopy using CP portal. In addition to ultrasonography, we had also performed MRI and further delineated the radiological abnormalities. We have mentioned in our manuscript that the lack of longitudinal observations was a limitation for our study and a later cross-sectional evaluation to delineate whether the radiological findings would differ by time seems to be

\footnotetext{
A. Bayar $(\bowtie)$

Department of Orthopaedics and Traumatology, Faculty of Medicine, Zonguldak Karaelmas University, 67600 Kozlu, Zonguldak, Turkey

e-mail: bayarahmet@yahoo.com
}

appropriate. Thus, we think that a cross-sectional study at 4-6 weeks postoperatively would not be sufficient to be sure that puncture of the tendon leaves nothing behind. As far as we know such prospective study is lacking and we do not clearly know the time of occurrence of fibrosis, scar tissue, granulation tissue or mucoid degeneration within the tendon. On the other hand, the manner of use of the portal might cause some difference. In our practice, we had used the portal interchangeably for holding and cutting instruments and the telescope: i.e. the tendon was penetrated a few times through this portal during the procedure. If the tendon was got across only once for the placement of a particular instrument, the harm to the tendon might be expected to be reduced.

Finally, we had stated that use of CP portal and accidental violation of fat pad did not produce any clinical problems in a low demand group of patients. Although we agree with the authors of the letter that, interpretation of changes strictly as an abnormality in the tendon might be confusing, the significant radiological sequela in our patients could not be overlooked. Rather than concluding that the portal is unsafe, we preferred to state that it was not as safe as generally thought to be and we feel that until these changes were interpreted with biomechanical studies caution should be held in patients who are or may be candidates for ACL reconstruction.

\section{Reference}

1. Bayar A, Turhan E, Özer T, Keser S, Ege A, Erdem Z (2008) The fate of patellar tendon and infrapatellar fat pad after arthroscopy via central portal. Knee Surg Sports Traumatol Arthrosc 16:11141120. doi:10.1007/s00167-008-0612-0 